\title{
Crystal structure of diaqua-bis[(5-nitroisophthalato)-(4,4'- dipyridylsulfide)cobalt(II)], $\mathrm{Co}_{2}\left(\mathrm{H}_{2} \mathrm{O}\right)_{2}\left(\mathrm{C}_{8} \mathrm{H}_{3} \mathrm{NO}_{6}\right)_{2}\left(\mathrm{C}_{10} \mathrm{H}_{8} \mathrm{~N}_{2} \mathrm{~S}\right)_{2}$
}

\author{
Shuai Yuan, Wen-Ying Zhang*, Kun-Xian Shu, Yi-Long Liang and Guang-Fan Yan \\ Chongqing University of Post and Telecommunication, College of Bioinformation, Chongqing 400065, P. R. China
}

Received December 30, 2008, accepted and available on-line February 27, 2009; CCDC no. 1267/2535

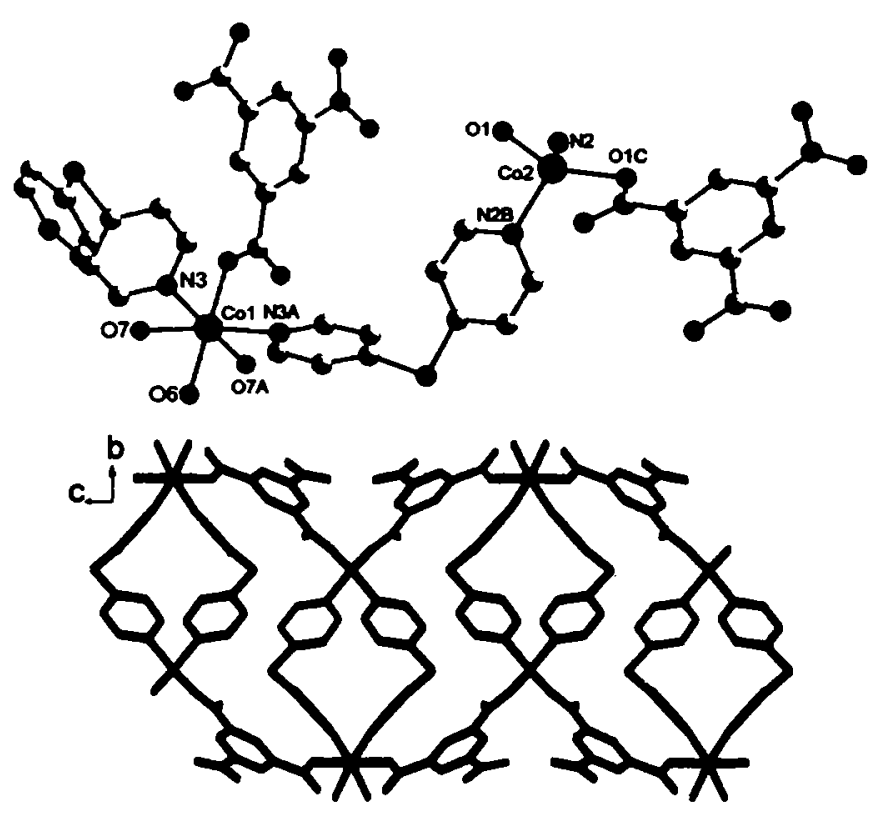

Abstract

$\mathrm{C}_{72} \mathrm{H}_{52} \mathrm{Co}_{4} \mathrm{~N}_{12} \mathrm{O}_{28} \mathrm{~S}_{4}$, monoclinic, $P 2 / c$ (no. 13), $a=14.120(2) \AA, b=8.4612(9) \AA, c=20.550(2) \AA$, $\beta=129.470(5)^{\circ}, V=1895.3 \AA^{3}, Z=1, R_{g t}(F)=0.028$, $w R_{\text {ref }}\left(F^{2}\right)=0.077, T=291 \mathrm{~K}$.

\section{Source of material}

A mixture of 5-nitroisophthalic acid $\left(\mathrm{O}_{2} \mathrm{~N}-\mathrm{H}_{2} \mathrm{ip}, 0.1 \mathrm{mmol}, 20.5\right.$ $\mathrm{mg}$ ), 4,4'-bipyridyl sulfide (bps, $0.1 \mathrm{mmol}, 18.9 \mathrm{mg}), \mathrm{Co}(\mathrm{OAc})_{2}$ $4 \mathrm{H}_{2} \mathrm{O}(24.7 \mathrm{mg}, 0.1 \mathrm{mmol}), \mathrm{KOH}(0.1 \mathrm{mmol}, 5.5 \mathrm{mg})$ and $\mathrm{H}_{2} \mathrm{O} 18$ $\mathrm{ml}$ was placed in a Teflon-lined stainless steel vessel, heated to $180^{\circ} \mathrm{C}$ for 3 days, and then cooled to room temperature over $48 \mathrm{~h}$. Red block-shaped crystals of the title compound were obtained.

\section{Discussion}

The design of metal-organic coordination polymers is of current interest in the fields of supramolecular chemistry and crystal engineering. Major reasons for this interest stem from the intriguing variety of topologies and structural diversity, such as helixes and diamondoid nets, and because of their potential applications as functional materials, such as hetero-geneous catalysis, molecular recognition, magnetism, gas storage, ion exchange, nonlinear optics, and electrical conductivity $[1,2]$. It is well known that organic ligands play crucial roles in the design and construction of desirable frameworks. In this context, benzenedicarboxylic acid and their derivatives (such as 1,3-benzenedicarboxylic acid, 1,4

benzenedicarboxylic acid, 5-nitroisophthalic acid, 5-hydroxyisophtalic acid etc.) are widely used as building blocks to link metal ions to produce interesting metal-organic framework structures and properties $[3,4]$. On the other hand, in contrast to the large and increasing amount of work on the synthesis with ligands containing $\mathbf{O}$ or $\mathbf{N}$ donors, there have been fewer reports of studies based on organothiolate ligands. 4,4'-Dipyridyl sulfide (dps) possesses a non-linear backbone due to the bend around the sulfur atom, and may yield various coordination polymers [5].

The fundamental building unit of the title crystal structure contains two cobalt ions, two $\mathrm{O}_{2} \mathrm{~N}$-ip ligands, two bps ligands and two coordinated water molecules (figure, top). The $\mathrm{Col}$ ion is 6coordinated with a distorted octahedral environment. The six atoms coordinated to each $\mathrm{Co}$ (II) ion come from two carboxylate oxygen atoms from two $\mathrm{O}_{2} \mathrm{~N}$-ip ligands, two water molecules and two nitrogen atoms from two bps ligands. $\mathrm{Co} 2$ adopts a distorted tetrahedral $\left[\mathrm{N}_{2} \mathrm{O}_{2}\right]$ coordination, and is coordinated by two carboxylic oxygen atoms from two $\mathrm{O}_{2} \mathrm{~N}$-ip ligands and two bps nitrogen atoms. The $\mathrm{Co}-\mathrm{O}$ and $\mathrm{Co}-\mathrm{N}$ bond lengths are in the range of $1.978(2)-2.173(2) \AA$ and $2.059(2)-2.178(2) \AA$, respectively.

Every bps ligand adopts a bis-monodentate bridging mode and links neighboring $\mathrm{Co}$ (II) ions, resulting in a $1 \mathrm{D}$ chain. The 1D chains are further connected by $\mathrm{O}_{2} \mathrm{~N}$-ip ligands to give a puckered 2D sheet (figure, bottom). It is noteworthy that $\mathrm{O}_{2} \mathrm{~N}$-ip serves as not only coordinative ligands, but also weak-interaction donor and acceptor. It is interesting to observe that the adjacent 2D layers are connected by $\mathrm{O} \cdots \mathrm{O}$ intermolecular contacts $(d(\mathrm{O} 4 \cdots \mathrm{O})=$ $3.019 \AA$ ) between the uncoordinated carboxylate oxygens and the $-\mathrm{NO}_{2}$ groups of $\mathrm{O}_{2} \mathrm{~N}$-ip ligands, leading to a 3D supramolecular network.

Table 1. Data collection and handling.

Crystal:

Wavelength:

$\mu$ :

Diffractometer, scan mode:

$2 \theta_{\max }:$

$N(h k l)_{\text {measured, }} N(h k l)_{\text {mique: }}$

Criterion for $I_{\mathrm{obs}}, N(h k l)_{\mathrm{gt}}$ :

$N(\text { param })_{\text {refined: }}$

Programs: red block, size $0.07 \times 0.23 \times 0.39 \mathrm{~mm}$

Mo $K_{\alpha}$ radiation $(0.71073 \AA)$

$10.64 \mathrm{~cm}^{-1}$

Bruker SMART CCD, $\varphi / \omega$

$51^{\circ}$

13968, 3533

$I_{\text {obs }}>2 \sigma\left(I_{o b s}\right), 2876$

272

SHELXS-97 [6], SHELXL-97 [7], SHELXTL [8]

* Correspondence author (e-mail: zhangwy_hi@126.com) 
Table 2. Atomic coordinates and displacement parameters (in $\AA^{2}$ ).

\begin{tabular}{llllll}
\hline Atom & Site & $x$ & $y$ & $z$ & \multicolumn{1}{l}{$U_{\text {iso }}$} \\
\cline { 1 - 3 } H(1W) & $4 g$ & 0.4841 & 1.0250 & 0.3084 & 0.066 \\
H(2W) & $4 g$ & 0.3733 & 0.9474 & 0.2609 & 0.066 \\
H(3) & $4 g$ & 1.1615 & 0.5834 & 0.6731 & 0.036 \\
H(5) & $4 g$ & 1.0178 & 0.8625 & 0.4709 & 0.034 \\
H(7A) & $4 g$ & 0.7962 & 0.6191 & 0.4939 & 0.037 \\
H(9) & $4 g$ & 0.1264 & -0.0195 & 0.2276 & 0.051 \\
H(10) & $4 g$ & 0.2721 & 0.1737 & 0.2988 & 0.051 \\
\hline
\end{tabular}

Table 2. Continued.

\begin{tabular}{llllll}
\hline Atom & Site & $x$ & $y$ & $z$ & \multicolumn{1}{l}{$U_{\text {iso }}$} \\
\hline $\mathrm{H}(12)$ & $4 g$ & 0.3201 & 0.0529 & 0.5065 & 0.053 \\
$\mathrm{H}(13)$ & $4 g$ & 0.1756 & -0.1398 & 0.4298 & 0.052 \\
$\mathrm{H}(15)$ & $4 g$ & 0.2793 & 0.5084 & 0.3503 & 0.045 \\
$\mathrm{H}(16)$ & $4 g$ & 0.3025 & 0.6684 & 0.2710 & 0.042 \\
$\mathrm{H}(17)$ & $4 g$ & 0.6104 & 0.4650 & 0.3597 & 0.047 \\
$\mathrm{H}(18)$ & $4 g$ & 0.5970 & 0.2982 & 0.4420 & 0.048 \\
& & & & & \\
\hline
\end{tabular}

Table 3. Atomic coordinates and displacement parameters (in $\AA^{2}$ ).

\begin{tabular}{|c|c|c|c|c|c|c|c|c|c|c|}
\hline Atom & Site & $x$ & $y$ & $z$ & $U_{11}$ & $U_{22}$ & $U_{33}$ & $U_{12}$ & $U_{13}$ & $U_{23}$ \\
\hline $\operatorname{Co}(1)$ & $2 f$ & $1 / 2$ & $0.75690(5)$ & $1 / 4$ & $0.0243(2)$ & $0.0321(2)$ & $0.0227(2)$ & $\mathbf{0}$ & $0.0086(2)$ & 0 \\
\hline $\operatorname{Co}(2)$ & $2 e$ & 0 & $-0.26464(4)$ & $1 / 4$ & $0.0292(2)$ & $0.0259(2)$ & $0.0266(2)$ & 0 & $0.0174(2)$ & 0 \\
\hline $\mathbf{S}(1)$ & $4 g$ & $0.43419(6)$ & $0.26976(8)$ & $0.47725(4)$ & $0.0513(4)$ & $0.0521(4)$ & $0.0307(3)$ & $-0.0255(3)$ & $0.0208(3)$ & $-0.0085(3)$ \\
\hline$O(1)$ & $4 g$ & $1.0504(1)$ & $0.3804(2)$ & $0.69275(9)$ & $0.0389(9)$ & $0.0464(9)$ & $0.0369(8)$ & $0.0036(7)$ & $0.0207(8)$ & $0.0180(7)$ \\
\hline$O(2)$ & $4 g$ & $0.8566(2)$ & $0.4496(2)$ & $0.6184(1)$ & $0.050(1)$ & $0.059(1)$ & $0.080(1)$ & $0.0070(9)$ & $0.047(1)$ & $0.028(1)$ \\
\hline$O(3)$ & $4 g$ & $1.3144(1)$ & $0.7522(2)$ & $0.6892(1)$ & $0.0266(8)$ & $0.053(1)$ & $0.0343(9)$ & $0.0039(7)$ & $0.0122(7)$ & $0.0081(7)$ \\
\hline O(4) & $4 g$ & $1.2465(2)$ & $0.8467(2)$ & $0.5688(1)$ & $0.044(1)$ & $0.074(1)$ & $0.048(1)$ & $-0.0020(9)$ & $0.0348(9)$ & $0.0090(9)$ \\
\hline$O(5)$ & $4 g$ & $0.7903(1)$ & $0.9044(2)$ & $0.34383(9)$ & $0.0358(8)$ & $0.0410(9)$ & $0.0286(8)$ & $-0.0006(7)$ & $0.0149(7)$ & $0.0101(7)$ \\
\hline$O(6)$ & $4 g$ & $0.6760(1)$ & $0.7546(2)$ & $0.35992(9)$ & $0.0270(8)$ & $0.052(1)$ & $0.0300(8)$ & $-0.0071(7)$ & $0.0063(7)$ & $0.0121(7)$ \\
\hline$O(7)$ & $4 g$ & $0.4497(2)$ & $0.9417(2)$ & $0.2964(1)$ & $0.052(1)$ & $0.044(1)$ & $0.0404(9)$ & $0.0059(8)$ & $0.0162(8)$ & $-0.0089(8)$ \\
\hline $\mathrm{N}(1)$ & $4 g$ & $1.2306(2)$ & $0.7805(2)$ & $0.6145(1)$ & $0.0296(9)$ & $0.036(1)$ & $0.034(1)$ & $0.0037(7)$ & $0.0206(9)$ & $0.0026(8)$ \\
\hline $\mathbf{N}(2)$ & $4 g$ & $0.1370(2)$ & $-0.0994(2)$ & $0.3213(1)$ & $0.038(1)$ & $0.0298(9)$ & $0.0288(9)$ & $-0.0066(8)$ & $0.0209(8)$ & $-0.0040(7)$ \\
\hline $\mathbf{N}(3)$ & $4 g$ & $0.4599(2)$ & $0.5851(2)$ & $0.3085(1)$ & $0.0272(9)$ & $0.0334(9)$ & $0.0292(9)$ & $-0.0031(7)$ & $0.0156(8)$ & $-0.0047(7)$ \\
\hline$C(1)$ & $4 g$ & $0.9576(2)$ & $0.4632(2)$ & $0.6364(1)$ & $0.040(1)$ & $0.032(1)$ & $0.031(1)$ & $-0.0026(9)$ & $0.022(1)$ & $0.0019(9)$ \\
\hline$C(2)$ & $4 g$ & $0.9755(2)$ & $0.5810(2)$ & $0.5902(1)$ & $0.033(1)$ & $0.027(1)$ & $0.026(1)$ & $-0.0009(8)$ & $0.0177(9)$ & $0.0017(8)$ \\
\hline$C(3)$ & $4 g$ & $1.0928(2)$ & $0.6253(2)$ & $0.6225(1)$ & $0.028(1)$ & $0.032(1)$ & $0.023(1)$ & $0.0039(9)$ & $0.0129(9)$ & $0.0046(8)$ \\
\hline C(4) & $4 g$ & $1.1056(2)$ & $0.7329(2)$ & $0.5779(1)$ & $0.027(1)$ & $0.029(1)$ & $0.029(1)$ & $-0.0003(8)$ & $0.0170(9)$ & $-0.0016(8)$ \\
\hline$C(5)$ & $4 g$ & $1.0063(2)$ & $0.7938(2)$ & $0.5006(1)$ & $0.035(1)$ & $0.026(1)$ & $0.024(1)$ & $-0.0018(8)$ & $0.0178(9)$ & $0.0012(8)$ \\
\hline$C(6)$ & $4 g$ & $0.8889(2)$ & $0.7496(2)$ & $0.4688(1)$ & $0.029(1)$ & $0.026(1)$ & $0.023(1)$ & $-0.0007(8)$ & $0.0127(9)$ & $0.0008(8)$ \\
\hline$C(7)$ & $4 g$ & $0.8748(2)$ & $0.6458(2)$ & $0.5145(1)$ & $0.027(1)$ & $0.031(1)$ & $0.027(1)$ & $-0.0026(8)$ & $0.0139(9)$ & $0.0007(8)$ \\
\hline$C(8)$ & $4 g$ & $0.7769(2)$ & $0.8099(3)$ & $0.3837(1)$ & $0.031(1)$ & $0.031(1)$ & $0.023(1)$ & $-0.0011(9)$ & $0.0120(9)$ & $-0.0002(9)$ \\
\hline$C(9)$ & $4 g$ & $0.1668(2)$ & $-0.0054(3)$ & $0.2847(1)$ & $0.053(1)$ & $0.049(1)$ & $0.028(1)$ & $-0.016(1)$ & $0.027(1)$ & $-0.007(1)$ \\
\hline$C(10)$ & $4 g$ & $0.2541(2)$ & $0.1113(3)$ & $0.3270(1)$ & $0.053(1)$ & $0.046(1)$ & $0.036(1)$ & $-0.020(1)$ & $0.032(1)$ & $-0.009(1)$ \\
\hline$C(11)$ & $4 g$ & $0.3147(2)$ & $0.1348(2)$ & $0.4116(1)$ & $0.035(1)$ & $0.033(1)$ & $0.031(1)$ & $-0.0060(9)$ & $0.021(1)$ & $-0.0054(9)$ \\
\hline$C(12)$ & $4 g$ & $0.2827(2)$ & $0.0392(3)$ & $0.4499(1)$ & $0.057(2)$ & $0.044(1)$ & $0.025(1)$ & $-0.018(1)$ & $0.023(1)$ & $-0.007(1)$ \\
\hline$C(13)$ & $4 g$ & $0.1953(2)$ & $-0.0755(3)$ & $0.4032(1)$ & $0.056(1)$ & $0.042(1)$ & $0.032(1)$ & $-0.016(1)$ & $0.028(1)$ & $-0.003(1)$ \\
\hline$C(14)$ & $4 g$ & $0.4369(2)$ & $0.3858(2)$ & $0.4059(1)$ & $0.036(1)$ & $0.032(1)$ & $0.033(1)$ & $-0.0146(9)$ & $0.020(1)$ & $-0.0105(9)$ \\
\hline$C(15)$ & $4 g$ & $0.3478(2)$ & $0.4979(3)$ & $0.3536(1)$ & $0.028(1)$ & $0.046(1)$ & $0.036(1)$ & $-0.009(1)$ & $0.019(1)$ & $-0.009(1)$ \\
\hline$C(16)$ & $4 g$ & $0.3628(2)$ & $0.5938(3)$ & $0.3063(1)$ & $0.025(1)$ & $0.040(1)$ & $0.030(1)$ & $-0.0009(9)$ & $0.0129(9)$ & $-0.0030(9)$ \\
\hline$C(17)$ & $4 g$ & $0.5434(2)$ & $0.4746(3)$ & $0.3583(1)$ & $0.036(1)$ & $0.036(1)$ & $0.050(1)$ & $0.003(1)$ & $0.029(1)$ & $0.000(1)$ \\
\hline$C(18)$ & $4 g$ & $0.5362(2)$ & $0.3732(3)$ & $0.4080(2)$ & $0.037(1)$ & $0.032(1)$ & $0.047(1)$ & $0.004(1)$ & $0.025(1)$ & $0.005(1)$ \\
\hline
\end{tabular}

Acknowledgment. This research is supported by Chongqing University of Posts and Telecommunications Youth Fund (A2006-50).

\section{References}

1. Cao, R.; Sun, D. F.; Liang, Y. C.; Hong, M. C.; Tatsumi, K.; Shi, Q.: Syntheses and Characterizations of Three-Dimensional Channel-like Polymeric Lanthanide Complexes Constructed by 1,2,4,5-Benzenetetracarboxylic Acid. Inorg. Chem. 41 (2002) 2087-2094.

2. Luo, F.; Hu, D. X.; Che, Y. X.; Zheng, J. M.: Pillared 3d-4f Frameworks with Rare 3D Architecture Showing the Coexistence of Ferromagnetic and Antiferromagnetic Interactions between Gadolinium Ions. Cryst. Growth Des. 7 (2007) $851-853$.

3. Ma, L. F.; Wang, L. Y.; Hu, J. L.; Wang, Y. Y.; Batten, S. R.; Wang, J. G.: Synthesis, structures and properties of $\mathrm{Mn}(\mathrm{II})$ metal organic frameworks based on $\mathrm{R}$-isophthalate $\left(\mathrm{R}=\mathrm{CH}_{3}, \mathrm{C}\left(\mathrm{CH}_{3}\right)_{3}\right)$ and different pyridylcontaining ligands. Cryst. Eng. Comm. 11 (2009) 109-117.
4. Ma, L. F.; Wang, L. Y.; Wang, Y. Y.; Batten, S. R.; Wang, J. G.: SelfAssembly of a Series of Cobalt(II) Coondination Polymers Constructed from $\mathrm{H}_{2}$ tbip and Dipyridyl-Based Ligands. Inorg. Chem. 48 (2009) 915924.

5. Ma, L. F.; Wang, L. Y.; Hu, J. L.; Wang, Y. Y.; Batten, S. R.; Wang, J. G.: Dicarboxylate anion-dependent assembly of $\mathrm{Ni}(\mathrm{I})$ coordination polymers with 4,4'-dipyridyl sulfide. CrystEngComm 11 (2009) 777-783.

6. Sheldrick, G. M.: SHELXS-97. Program for the Solution of Crystal Structures. University of Göttingen, Germany 1997.

7. Sheldrick, G. M.: SHELXL-97. Program for the Refinement of Crystal Structures. University of Göttingen, Germany 1997.

8. Sheldrick, G. M.: SHELXTL. Structure Determination Software Suite. Version 6.14. Bruker AXS, Madison, Wisconsin, USA 2000. 